\title{
Renoprotective effects of berberine as adjuvant therapy for hypertensive patients with type 2 diabetes mellitus: Evaluation via biochemical markers and color Doppler ultrasonography
}

\author{
PEIFENG DAI ${ }^{1}$, JUNHUA WANG ${ }^{1}$, LIN LIN $^{2}$, YANYAN ZHANG $^{3}$ and ZHENGPING WANG ${ }^{1}$ \\ Departments of ${ }^{1}$ Ultrasound and ${ }^{2}$ Nephrology, Weifang People's Hospital, Weifang, Shandong 261041; \\ ${ }^{3}$ Department of Rheumatology and Immunology, The Affiliated Hospital of Shandong \\ University of Traditional Chinese Medicine, Jinan, Shandong 250011, P.R. China
}

Received August 16, 2014; Accepted May 13, 2015

DOI: 10.3892/etm.2015.2585

\begin{abstract}
Diabetes and hypertension are complex and serious diseases that may ultimately lead to renal complications. Adequate control of blood glucose and blood pressure contributes to decreased renal risks, but may not be sufficient for certain patients. The current study was undertaken to investigate the renoprotective effects of berberine as an adjuvant therapy to standard hypotensive and hypoglycemic treatment in hypertensive patients with type 2 diabetes mellitus (T2DM). In this 2-year clinical study, 69 hypertensive patients with T2DM, whose blood pressure and fasting plasma glucose (FPG) were adequately controlled by hypotensive and oral hypoglycemic agents prior to the study, were enrolled and randomly assigned into control (33 cases) and add-on (36 cases) groups. Berberine was orally administrated to the patients in the add-on group concomitantly with standard hypotensive and hypoglycemic treatment. Baseline characteristics, including the levels of FPG, glycated hemoglobin, systolic blood pressure, diastolic blood pressure, serum creatinine, urinary albumin-to-creatine ratio (UACR), urinary osteopontin and kidney injury molecule-1 (KIM-1) were determined. Furthermore, the oxidative stress markers malondialdehyde, urinary 8-hydroxy-2'-deoxyguanosine, superoxide dismutase, glutathione peroxidase and total-antioxidant capacity, and the inflammatory parameters vascular adhesion molecule-1, C-reactive protein and high molecular weight-adiponectin were evaluated. In addition, ultrasonographic parameters, including peak systolic velocity, end diastolic velocity and renal arterial resistance index were determined. After treatment, it was observed that the control and add-on treatments were able to
\end{abstract}

Correspondence to: Dr Junhua Wang, Department of Ultrasound, Weifang People's Hospital, 151 Guangwen Road, Weifang, Shandong 261041, P.R. China

E-mail: wangjunhuawf@126.com

Key words: hypertension, type 2 diabetes mellitus, berberine, add-on therapy, renoprotective effect, color Doppler ultrasonography adequately control blood pressure and blood glucose. Patients in the add-on group exhibited significant reductions in renal damage biochemical markers (UACR, urinary osteopontin and KIM-1) and improved renal hemodynamics, in addition to reduced inflammation and oxidative stress. The present results suggest that berberine is beneficial for hypertensive patients with T2DM as add-on therapy to standard hypotensive and hypoglycemic agents.

\section{Introduction}

Hypertension and diabetes are serious chronic diseases that affect millions of individuals worldwide and increase risks of cardiovascular events and renal failure (1). Furthermore, it is well demonstrated that hypertension complicated with type 2 diabetes (T2DM) leads to an increased incidence of cardiovascular events $(2,3)$. Therefore, it is crucial to consider the risk of cardiovascular complications in hypertensive patients with T2DM.

Adequate glycemic and blood pressure control are believed to contribute to decreased risk of cardiovascular complications, including renal vascular damage. Previous clinical trials and animal studies have indicated that angiotensin-converting enzyme inhibitors (ACEIs) and long-acting calcium channel blockers are beneficial for the control of blood pressure and cardiovascular remodeling in hypertensive patients $(4,5)$. Furthermore, certain oral hypoglycemic drugs, such as metformin and gliclazide, have been demonstrated to be contributory to the control of cardiovascular remodeling (6). However, numerous patients with one or two of the conditions continue to exhibit various cardiovascular complications, including hypertensive nephropathy (HN) and diabetic nephropathy (DN), despite possessing adequately controlled blood pressure and blood glucose. This result may be due to the complex mechanisms leading to $\mathrm{HN}$ and $\mathrm{DN}$, which involve factors other than high levels of blood pressure and blood glucose.

Among the most common pathological symptoms of hypertension and diabetes is renal atherosclerosis, which may lead to renal failure. Inflammation has been well demonstrated to be crucially involved in the initiation and progression of renal atherosclerosis (7). Simultaneously, it is generally accepted 
that hypertension and diabetes are inflammatory conditions. Previous studies suggest that the levels certain inflammatory factors, such as vascular cell adhesion molecule 1 (VCAM-1) and C-reactive protein (CRP), are increased in patients with hypertension and diabetes $(8,9)$. Elevated inflammatory factors are hypothesized to be involved in the pathogenesis of renal damage, and treatments targeted at controlling these factors have shown therapeutic effects on cardiovascular complications. Furthermore, oxidative stress serves a crucial function in the pathogenesis of nephropathy induced by hypertension or diabetes $(10,11)$. Patients with hypertension or diabetes typically exhibit elevated oxidative stress activity, thus resulting in renal damage. Antioxidative treatment has exerted renoprotective effects in hypertensive and diabetic subjects in previous clinical and animal studies (10,12-15). Certain hypotensive drugs and oral hypoglycemic drugs, such as ACEIs, angiotensin II receptor blockers, metformin and gliclazide, exert various pharmacological actions, with anti-inflammatory and antioxidative effects $(12,16)$. However, studies indicate that inflammation and oxidative stress cannot be adequately controlled using these medications alone (17-23). Drugs possessing anti-inflammatory and antioxidative effects appear to be necessary for hypertensive patients with T2DM who are at high risk of vascular complications.

Berberine, a Traditional Chinese Medicine, has been reported to have various pharmacological effects and has been used to treat a variety of diseases, including cardiovascular disorders, inflammatory diseases and oxidative states. Li et al (24) reported that berberine improved pressure overload-induced cardiac hypertrophy and dysfunction in rats. A study by Zhao et al (25) indicated that berberine was able to improve the cardiac contractility and inhibit left ventricular remodeling in a rat model of renovascular hypertension, established by the two-kidney, two-clip method. Similarly, Hong et al (26) observed that berberine was able to improve abnormal cardiac function and prevent the development of left ventricular hypertrophy in rats. In addition, Cheng et al (27) reported that berberine improved endothelial function by reducing oxidative stress in humans. Furthermore, studies have demonstrated that berberine exhibits antioxidative and anti-inflammatory activity in diabetic subjects and berberine has been used to manage diabetes and its various complications (28-32). However, to the best of our knowledge, the renoprotective effects of berberine in hypertensive patients with T2DM remain unclear.

Certain biochemical markers, such as blood urea nitrogen, creatinine and urinary albumin, are typically used to assess renal dysfunction, and recently urinary osteopontin and kidney injury molecule-1 (KIM-1) have also been used as specific biomarkers of the early-stage renal damage (33). However, laboratory detections primarily demonstrate functional abnormalities of the kidneys, which indirectly reflect renal atherosclerosis. Ultrasonographic investigation, a noninvasive diagnostic procedure, is able to directly display certain characteristics of renal atherosclerosis, which are regarded as potential indicators for monitoring the progression of renal damage (34). Peak systolic velocity (PSV), end diastolic velocity (EDV), mean velocity and the acceleration time of the interlobar renal artery, as well as the renal arterial resistance index (RI) and pulsatility index (PI) are well documented parameters for the evaluation of renal hemodynamics and renal function $(35,36)$. Thus, the present study was undertaken to investigate the renoprotective effects of berberine, determined via laboratory and ultrasonographic analysis, as an add-on therapy to hypotensive and hypoglycemic medications in hypertensive patients with T2DM, in addition to the effects of berberine on a number of inflammatory and oxidative parameters.

\section{Materials and methods}

Participants. A study population of 69 patients with hypertension and T2DM were enrolled in the study. Patient blood pressure and fasting plasma glucose (FPG) were adequately controlled using hypotensive drugs and oral hypoglycemic agents prior to the study. The inclusion criteria for the patients were as follows: i) Systolic blood pressure (SBP) $<140 \mathrm{mmHg}$, diastolic blood pressure (DBP) $<90 \mathrm{mmHg}$ and FPG $<6.1 \mathrm{mmol} / \mathrm{l}$ when treated; ii) treatment prior to the study consisted of hypotensive agents (benazepril and amlodipine) and oral hypoglycemic drugs (metformin and gliclazide) and had been used for $>2$ years; iii) urinary albumin-to-creatine ratio (UACR), 30-200 $\mu \mathrm{g} / \mathrm{mg}$; and iv) age, $40-70$ years. The major exclusion criteria were as follows: i) Any type of diabetes mellitus other than T2DM; ii) secondary hypertension; iii) any other renal diseases; iv) any one of the following that might increase the risk of withdrawal from the study due to the severity and complexity of the conditions: history of myocardial infarction, stroke or coronary artery bypass grafting within 6 months prior to consent; class III or IV heart failure; unstable angina pectoris; other serious conditions. The protocols used in this study were approved by the Research Ethics Committee of Weifang People's Hospital (Weifang, China). Written informed consent was obtained from all patients prior to the initiation of the study.

Treatment. The 69 patients involved in this study were allocated at random into control (33 cases) and add-on (36 cases) groups. Subjects in the control group received the hypotensive and hypoglycemic baseline treatment in order to adequately control blood pressure and blood glucose, which consisted of benazepril (Nuohua Pharmaceutical Co. Ltd, Beijing, China), amlodipine (Pfizer Dalian Co., Dalian, China), metformin (Qingdao Huanghai Pharmaceutical Co. Ltd., Qingdao, China) and gliclazide (Beijing Wansheng Pharmaceutical Co. Ltd., Beijing, China), and was comparable with the background treatment. Patients in the add-on group were orally administered berberine (Shandong Renhetang Pharmaceutical Co., Ltd., Shandong, China) for 24 months in addition to the baseline treatment. The dose consisted of $0.1 \mathrm{~g}$ berberine, three times per day, with a 2 -week no-treatment interval every 5 months. There were no statistically significant differences in the mean doses of the drugs between the control and add-on groups.

Assessment of blood pressure and blood glucose. Blood pressure was measured between 8:00 and 9:00 a.m. by the same investigator. FPG and glycated hemoglobin $\left(\mathrm{HbA}_{1 \mathrm{c}}\right)$ levels were detected after a 12-h overnight fast.

Assessment of biochemical markers of renal damage. Concentrations of creatinine in freshly collected blood and 
urine samples were detected using an automatic biochemistry analyzer (Hitachi 7600; Hitachi, Tokyo, Japan). Urinary albumin was measured using chemiluminescence analysis (Fujirebio Diagnostics, Inc., Tokyo, Japan). Levels of urinary osteopontin and KIM-1, specific biomarkers of the early-stage renal damage, were measured using commercially available ELISA kits according to the instructions provided by the manufacturer (Wuhan Boster Biological Technology Ltd., Wuhan, China). Briefly, $100 \mu 1$ standards or sample were added per well and incubated for $2 \mathrm{~h}$ at $37^{\circ} \mathrm{C}$ with wells covered by the adhesive strip. Liquid was removed from the wells and $100 \mu \mathrm{l}$ biotin-conjugated monoclonal primary antibody solution (mouse anti-human, 1:100) was added to each well and incubated for $1 \mathrm{~h}$ at $37^{\circ} \mathrm{C}$. Next, $100 \mu \mathrm{l}$ avidin-peroxidase complex was added, after the wells had been washed, and the plate was incubated for $30 \mathrm{~min}$. Then, 3,3',5,5'-tetramethylbenzidine solution was added, the plate was maintained in the dark for $15 \mathrm{~min}$, and then $50 \mu 1$ stop solution was added to each well. Optical density at $450 \mathrm{~nm}$ was determined immediately, using a SpectraMax M5 microplate reader (Molecular Devices, Sunnyvale, CA, USA), and the concentrations of the markers were calculated.

Assessment of oxidative stress. Antioxidative parameters, including superoxide dismutase (SOD), glutathione peroxidase (GSH-Px) and total-antioxidant capacity (T-AOC), and the oxidative parameter malondialdehyde (MDA) in serum were evaluated using commercially available kits, in strict accordance with the manufacturer's instructions (Nanjing Jiancheng Bioengineering Institute, Nanjing, China) as previously described (37,38). The levels of urinary 8-hydroxy-deoxyguanosine (U-8-OHdG), a novel oxidative stress marker, were evaluated using an ELISA assay (Bioss Biosynthesis Biotechnology Co., Ltd., Beijing, China) according to the manufacturer's instructions.

Assessment of inflammatory parameters. Inflammation serves a crucial function in the pathogenesis of $\mathrm{HN}$ and DN; therefore, the levels of inflammatory parameters were measured. Serum VCAM-1 was measured by ELISA (Bender MedSystems $\mathrm{GmbH}$, eBioscience Corporation, San Diego, CA, USA). CRP was quantified using an automatic biochemical analyzer (Bio-Rad Laboratories, Inc., Benicia, CA, USA). Serum high-molecular-weight adiponectin (HMW-APN) levels were investigated using a chemiluminescent enzyme immunoassay (Fujirebio Diagnostics, Inc.).

Ultrasonographic measurements. Ultrasonographic measurements were performed by the same three investigators who had been well trained prior to the study, as previously reported (36). Ultrasonography was used to measure the PSV and EDV of the interlobar renal artery, in addition to the maximal width, depth and length of the kidney. The renal arterial RI was calculated as [(PSV-EDV)/PSV] (39). The results from three measurements obtained from three separate vessels were averaged. Renal volume was calculated as one-half the product of the three dimensions of the kidney.

Statistical analysis. All analyses of the outcomes were conducted under the intention-to-treat principle. All data were analyzed using SPSS software, version 16.0 (SPSS, Inc., Chicago, IL, USA). Quantitative data are expressed as the mean \pm standard deviation, intergroup differences were evaluated by unpaired t-test. Qualitative data analyses were conducted using the $\chi^{2}$ test. $\mathrm{P}<0.05$ was considered to indicate a statistically significant result.

\section{Results}

Baseline characteristics of the patients. A study population of 69 patients with hypertension and T2DM were enrolled, and allocated at random into control and add-on groups. Baseline characteristics of the patients are presented in Table I. No significant differences in the baseline characteristics were observed between the two groups at the initiation of the study ( $P>0.05)$, which indicated that the two groups were comparable. No patients withdrew from the study throughout the study period.

Blood pressure and blood glucose control. In order to investigate whether the add-on treatment was able to adequately control blood pressure, SBP and DBP were measured at the initiation of the study and following treatment. Patients in the add-on group exhibited a SBP of $120 \pm 11 \mathrm{mmHg}$ and a DBP of $81 \pm 8 \mathrm{mmHg}$, which are similar to the values of patients in the control group $(121 \pm 14$ and $83 \pm 7 \mathrm{mmHg}$, respectively; $\mathrm{P}>0.05)$ at the start of the study. After the 2-year treatment, no marked differences were detected in SBP $(125 \pm 14$ and $127 \pm 15 \mathrm{mmHg}$, respectively; $\mathrm{P}>0.05)$ and $\mathrm{DBP}(83 \pm 9$ and $86 \pm 6 \mathrm{mmHg}$, respectively; $\mathrm{P}>0.05$ ) between the add-on and control groups. Furthermore, levels of FPG and $\mathrm{HbA}_{\mathrm{lc}}$ were comparable between the two groups prior to and following the 2-year treatment $(\mathrm{P}>0.05)$. These results suggest that both treatments were able to adequately control the blood pressure and the blood glucose of the patients (Table II).

Effects on biomarkers of renal damage. After 2 years of treatment, no marked differences were detected in the change of serum creatinine levels during the study between the add-on and control groups $(-1.05 \mathrm{mmol} / \mathrm{l} \mathrm{vs}$. $+2.02 \mathrm{mmol} / \mathrm{l}$, respectively; P>0.05). However, a significant reduction in UACR was observed in the add-on group compared with the control group $(-9.96$ vs. $+21.86 \mu \mathrm{g} / \mathrm{mg}$; $\mathrm{P}<0.05)$. Levels of urinary osteopontin and KIM-1, which are specific biomarkers of early-stage renal damage, were investigated. The results show a marked reduction in the levels of osteopontin and KIM-1 in the add-on group compared with the control group ( -93.19 vs. $+56.02 \mathrm{pg} / \mathrm{ml}$ and -40.79 vs. $+31.62 \mathrm{pg} / \mathrm{ml}$, respectively; $\mathrm{P}<0.05)$. The data indicate that renal function significantly improved in the add-on group compared with the control group (Table III).

Effects on oxidative stress. Oxidative stress is crucially involved in the pathogenesis of $\mathrm{HN}$ and DN. Results relating to the evaluation of serum oxidative stress are presented in Table IV. Significant reductions in MDA and U-8-OHdG values occurred in the add-on group compared with the control group (MDA, -2.68 vs. $+1.06 \mathrm{nmmol} / \mathrm{ml}$; U-8-OHdG, -2.89 vs. $+1.47 \mathrm{ng} / \mathrm{mg}$ creatinine, respectively; $\mathrm{P}<0.05)$. By contrast, the levels of SOD, GSH-Px and T-AOC increased significantly in the add-on group compared with the control group (SOD, +19.13 vs. $-5.53 \mathrm{U} / \mathrm{ml}$; GSH-Px, +35.17 vs. $-7.8 \mathrm{U} / \mathrm{l}$; T-AOC, +0.38 vs. $-0.26 \mathrm{U} / \mathrm{ml}$; respectively; $\mathrm{P}<0.05$ ). 
Table I. Baseline characteristics of the patients.

\begin{tabular}{|c|c|c|}
\hline Characteristics & Control group & Add-on group \\
\hline Patients (n) & 33 & 39 \\
\hline Age (years) & $53.06 \pm 10.36$ & $55.31 \pm 11.79$ \\
\hline Male (n) & 16 & 19 \\
\hline Weight (kg) & $65.35 \pm 15.36$ & $63.57 \pm 12.63$ \\
\hline Body mass index $\left(\mathrm{kg} / \mathrm{m}^{2}\right)$ & $24.10 \pm 4.36$ & $24.5 \pm 4.01$ \\
\hline FPG $(\mathrm{mmol} / \mathrm{l})$ & $5.85 \pm 1.16$ & $5.91 \pm 1.24$ \\
\hline $\mathrm{HbA}_{1 \mathrm{c}}(\%)$ & $6.72 \pm 1.15$ & $6.65 \pm 1.01$ \\
\hline $\mathrm{SBP}(\mathrm{mmHg})$ & $121 \pm 14$ & $120 \pm 11$ \\
\hline DBP (mmHg) & $83 \pm 7$ & $81 \pm 8$ \\
\hline UACR $(\mu \mathrm{g} / \mathrm{mg})$ & $65.18 \pm 8.52$ & $63.33 \pm 8.17$ \\
\hline Serum creatinine (mmol/l) & $52.13 \pm 7.30$ & $51.50 \pm 7.05$ \\
\hline Urinary osteopontin (pg/ml) & $829.75 \pm 103.12$ & $838.41 \pm 130.08$ \\
\hline Urinary KIM-1 (pg/ml) & $298.14 \pm 12.64$ & $295.08 \pm 10.11$ \\
\hline $\mathrm{SOD}(\mathrm{U} / \mathrm{ml})$ & $56.16 \pm 8.21$ & $57.54 \pm 8.90$ \\
\hline GSH-Px (U/l) & $136.6 \pm 16.1$ & $139.2 \pm 15.7$ \\
\hline T-AOC (U/ml) & $2.06 \pm 0.35$ & $2.10 \pm 0.37$ \\
\hline MDA (nmmol/ml) & $9.03 \pm 1.25$ & $9.32 \pm 1.06$ \\
\hline U-8-OHdG (ng/mg creatinine) & $11.12 \pm 1.25$ & $11.25 \pm 1.39$ \\
\hline VCAM-1 (ng/ml) & $682.36 \pm 55.14$ & $693.63 \pm 61.57$ \\
\hline $\mathrm{CRP}(\mathrm{mg} / \mathrm{l})$ & $1.95 \pm 0.35$ & $1.93 \pm 0.29$ \\
\hline HMW-APN (mg/ml) & $5.01 \pm 0.89$ & $5.14 \pm 1.06$ \\
\hline Peak systolic velocity (cm/sec) & $22.53 \pm 3.10$ & $23.10 \pm 3.51$ \\
\hline Minimal diastolic velocity $(\mathrm{cm} / \mathrm{sec})$ & $8.61 \pm 1.23$ & $8.93 \pm 1.21$ \\
\hline RI & $0.71 \pm 0.04$ & $0.70 \pm 0.03$ \\
\hline Renal volume $\left(\mathrm{cm}^{3}\right)$ & $120.10 \pm 18.35$ & $119.04 \pm 16.17$ \\
\hline
\end{tabular}

Unless otherwise indicated, data are expressed as the mean \pm standard deviation. FPG, fasting plasma glucose; HbA $\mathrm{H}_{1 \mathrm{c}}$, glycated hemoglobin; SBP, systolic blood pressure; DBP, diastolic blood pressure; UACR, urine albumin-to-creatine ratio; KIM-1, kidney injury molecule-1; SOD, superoxide dismutase; GSH-Px, glutathione peroxidase; T-AOC, total-antioxidant capacity; MDA, malondialdehyde; U-8-OHdG, urinary 8-hydroxy-2'-deoxyguanosine; VCAM-1, vascular adhesion molecule-1; CRP, C-reactive protein; HMW-APN, high molecular weight adiponectin; RI, renal arterial resistance index.

Table II. Levels of blood pressure and blood glucose of the patients before and after treatment.

\begin{tabular}{lcccc}
\hline & \multicolumn{2}{c}{ Control group } & \multicolumn{2}{c}{ Add-on group } \\
\cline { 2 - 4 } Parameter & Year 0 & Year 2 & Year 0 & Year 2 \\
\hline FPG $(\mathrm{mmol} / \mathrm{l})$ & $5.85 \pm 1.16$ & $6.03 \pm 1.31$ & $5.91 \pm 1.24$ & $5.99 \pm 1.17$ \\
$\mathrm{HbA}_{1 \mathrm{c}}(\%)$ & $6.72 \pm 1.15$ & $6.91 \pm 1.37$ & $6.65 \pm 1.01$ & $6.51 \pm 1.09$ \\
$\mathrm{SBP}(\mathrm{mmHg})$ & $121 \pm 14$ & $127 \pm 15$ & $120 \pm 11$ & $125 \pm 14$ \\
$\mathrm{DBP}(\mathrm{mmHg})$ & $83 \pm 7$ & $86 \pm 6$ & $81 \pm 8$ & $83 \pm 9$ \\
\hline
\end{tabular}

Data are expressed as the mean \pm standard deviation. FPG, fasting plasma glucose; $\mathrm{HbA}_{1 c}$, glycated hemoglobin; SBP, systolic blood pressure DBP, diastolic blood pressure.

These results indicate that the add-on treatment significantly inhibited oxidative stress in patients (Table IV).

Effects on inflammatory parameters. VCAM-1, CRP and HMW-APN are hypothesized to be associated with renal damage. The results reveal more marked reductions in the levels of serum VCAM-1 and CRP, in addition to more notable elevations in serum HMW-APN levels in the add-on group compared with the control group (VCAM-1, -156.34 vs. +49.36 ng/ml; CRP, -0.35 vs. +0.27 mg/l; HMW-APN, +1.58 
Table III. Renal damage parameters of the patients after treatment.

\begin{tabular}{lcc}
\hline Parameter & Control group & Add-on group \\
\hline Serum creatinine $(\mathrm{mmol} / \mathrm{l})$ & $2.02 \pm 1.67$ & $-1.05 \pm 0.89$ \\
UACR $(\mu \mathrm{g} / \mathrm{mg})$ & $21.86 \pm 4.03$ & $-9.96 \pm 1.41^{\mathrm{a}}$ \\
Urinary osteopontin $(\mathrm{pg} / \mathrm{ml})$ & $56.02 \pm 9.35$ & $-93.19 \pm 11.92^{\mathrm{a}}$ \\
Urinary KIM-1 $(\mathrm{pg} / \mathrm{ml})$ & $31.62 \pm 7.01$ & $-40.79 \pm 9.30^{\mathrm{a}}$ \\
\hline
\end{tabular}

Changes from the baseline to the end of the study are expressed as the mean \pm standard deviation. ${ }^{\mathrm{a}} \mathrm{P}<0.05$ vs. the control group. UACR, urine albumin-to-creatine ratio; KIM-1, kidney injury molecule-1.

Table IV. Oxidative stress parameters of the patients after treatment.

\begin{tabular}{lrr}
\hline Parameter & Control group & Add-on group \\
\hline U-8-OHdG (ng/mg creatinine) & $1.47 \pm 0.21$ & $-2.89 \pm 0.37^{\mathrm{a}}$ \\
MDA (nmmol/ml) & $1.06 \pm 0.20$ & $-2.68 \pm 0.45^{\mathrm{a}}$ \\
SOD $(\mathrm{U} / \mathrm{ml})$ & $-5.53 \pm 0.84$ & $19.13 \pm 4.01^{\mathrm{a}}$ \\
GSH-Px $(\mathrm{U} / \mathrm{l})$ & $-7.80 \pm 1.02$ & $35.17 \pm 6.41^{\mathrm{a}}$ \\
T-AOC $(\mathrm{U} / \mathrm{ml})$ & $-0.26 \pm 0.04$ & $0.38 \pm 0.06^{\mathrm{a}}$
\end{tabular}

Changes from the baseline to the end of the study are expressed as the mean \pm standard deviation. ${ }^{\text {a }}<0.05$ vs. control group. U-8-OHdG; urinary 8-hydroxy-2'-deoxyguanosine; MDA, malondialdehyde; SOD, superoxide dismutase; GSH-Px, glutathione peroxidase; T-AOC, total-antioxidant capacity.

Table V. Inflammatory parameters of the patients.

\begin{tabular}{lrr}
\hline Parameter & Control group & Add-on group \\
\hline VCAM-1 $(\mathrm{ng} / \mathrm{ml})$ & $49.36 \pm 8.05$ & $-156.34 \pm 18.96^{\mathrm{a}}$ \\
CRP $(\mathrm{mg} / \mathrm{l})$ & $0.27 \pm 0.05$ & $-0.35 \pm 0.07^{\mathrm{a}}$ \\
HMW-APN $(\mathrm{mg} / \mathrm{ml})$ & $-0.26 \pm 0.01$ & $1.58 \pm 0.26^{\mathrm{a}}$ \\
\hline
\end{tabular}

Changes from the baseline to the end of the study are expressed as the mean \pm standard deviation. ${ }^{\text {a }}<0.05$ vs. the control group. VCAM-1; vascular adhesion molecule-1; CRP, C-reactive protein; HMW-APN, high molecular weight-adiponectin.

Table VI. Ultrasonographic parameters of the interlobar renal artery.

\begin{tabular}{lcc}
\hline Parameter & Control group & Add-on group \\
\hline PSV $(\mathrm{cm} / \mathrm{sec})$ & $-1.27 \pm 0.18$ & $4.15 \pm 0.62^{\mathrm{a}}$ \\
$\mathrm{EDV}(\mathrm{cm} / \mathrm{sec})$ & $-0.84 \pm 0.11$ & $1.83 \pm 0.30^{\mathrm{a}}$ \\
$\mathrm{RI}$ & $0.01 \pm 0.004$ & $-0.04 \pm 0.005^{\mathrm{a}}$ \\
Renal volume $\left(\mathrm{cm}^{3}\right)$ & $5.06 \pm 2.06$ & $3.29 . \pm 1.31$ \\
\hline
\end{tabular}

Changes from the baseline to the end of the study are expressed as the mean \pm standard deviation. ${ }^{\mathrm{a}} \mathrm{P}<0.05 \mathrm{vs}$. the control group. PSV, peak systolic velocity; EDV, end diastolic velocity; RI, renal arterial resistance index.

vs. $-0.26 \mathrm{mg} / \mathrm{ml}$; respectively; $\mathrm{P}<0.05)$. These results indicate that the add-on treatment significantly attenuates inflammatory conditions in patients with HN and DN compared with the control treatment (Table V).
Ultrasonographic measurements. The ultrasonographic measurement revealed significant differences between the add-on and control groups in the improvement of the PSV (+4.15 vs. $-1.27 \mathrm{~cm} / \mathrm{sec})$, the EDV ( +1.83 vs. $-0.84 \mathrm{~cm} / \mathrm{sec})$, and 
RI (-0.04 vs. +0.01 , respectively; $\mathrm{P}<0.05)$. However, no marked difference was observed in renal volume between the add-on and control groups $\left(+3.29\right.$ vs. $+5.06 \mathrm{~cm}^{3}$; Table VI).

\section{Discussion}

The aim of the present study was to evaluate the effects of the long-term use of berberine on renal damage as an add-on therapy to hypotensive and hypoglycemic medications for the treatment of hypertensive patients with T2DM. Laboratory and ultrasonographic analysis was employed; in addition the effects of the treatment on a number of inflammatory and oxidative parameters were determined.

Hypertension and diabetes may ultimately lead to serious chronic renal diseases, in which atherosclerosis of the renal arteries is a key pathological development. Furthermore, patients with hypertension and T2DM are particularly vulnerable to cardiovascular disease and mortality $(2,3,40)$. Due to lifestyle changes and population aging, co-incidence of the two conditions is increasing, resulting in increasing numbers of individuals at risk of nephropathy and thus of renal failure. Adequate regulation of blood glucose and blood pressure contribute to a reduced renal risk, and ACEIs are regarded as effective agents against DN. However, under certain clinical conditions these medications are not sufficient. Consistently, the 69 hypertensive patients with T2DM enrolled in the current study exhibited impaired renal function (baseline UACR, $>30 \mu \mathrm{g} / \mathrm{mg}$ ) in spite of the adequate controls of blood pressure and blood glucose induced by hypotensive and oral hypoglycemic drugs such as ACEIs. Therefore, comprehensive therapy targeting various pathogenic factors is required.

Among the complex mechanisms leading to $\mathrm{DN}$ or $\mathrm{HN}$, inflammation plays a key role, and anti-inflammation managements have been demonstrated to be effective against DN and $\mathrm{HN}$ (41). Increased levels of the inflammatory factors VCAM-1 and CRP are associated with the vascular complications of diabetes and hypertension $(8,9,42)$. Renoprotective effects have been exhibited by treatments that decrease these inflammatory factors (43-46). HMW-APN, an anti-inflammatory cytokine secreted by adipose tissue, exerts a protective effect against cardiovascular disease (47). Low levels of HMW-APN have been found to be associated with cardiovascular risk (48). Previously, Kopf et al (49) reported that urinary HMW-APN excretion may identify diabetic patients at an increased risk of undergoing progression of kidney disease. In the current study, the results indicated more marked reductions in the levels of serum VCAM-1 and CRP, but significantly increased serum HMW-APN levels in the add-on group compared with the control group after 2 years of treatment. These data indicate significantly improved renal function in the add-on group compared with the control group. The present results are consistent with previous studies that reported the anti-inflammatory effects of berberine $(50,51)$. On the basis of these prior studies, it is hypothesized that improvement of inflammatory conditions using the add-on therapy may be beneficial for the recovery of renal damage.

Oxidative stress is another factor that promotes the initiation and progression of renal damage in patients with hypertension and diabetes $(10,11)$. Antioxidative stress treatment has been suggested for such patients, and has exhibited beneficial effects in the subjects. In the present study, MDA and U-8-OHdG levels were significantly reduced, while SOD, GSH-Px and T-AOC values increased significantly in the add-on group compared with the control group after 2 years of treatment. These data indicate that the add-on therapy significantly inhibited oxidative activity and promoted antioxidative activity in the patients. Similarly, the antioxidative and renoprotective effects of berberine have been reported in previous studies $(52,53)$. Therefore, it is hypothesized that the suppression of oxidative stress by berberine as add-on therapy in the current study contributed to the control of renal damage.

In addition to the evaluation of the aforementioned inflammatory and oxidative parameters, the effects of add-on berberine therapy on biochemical markers of renal damage were evaluated. The changes in the serum levels of creatinine over the 2 years of the study were not significantly different between the two groups. However, a significant reduction in UACR was observed in the add-on group compared with the control group ( $-9.96 \mathrm{vs} .+21.86 \mu \mathrm{g} / \mathrm{mg}$ ), which indicated that the add-on therapy provided improved renoprotection compared with the control therapy. Urinary osteopontin and KIM-1 are specific biomarkers for early-stage renal urinary damage, and the evaluation of osteopontin and KIM-1 levels indicated improved renoprotection in the add-on group compared with the control group. Therefore, the results of the present analyses indicate that the co-administration of berberine is more effective compared with the control therapy alone.

However, these laboratory parameters primarily demonstrate functional abnormalities in the patients, which indirectly reflect the renal atherosclerosis. Ultrasonographic analysis has been used to provide more direct information about the characteristics of renal atherosclerosis, and the data obtained are hypothesized to be potential indicators of the progression of renal damage (34-36). In the current study, Doppler ultrasonography was used to evaluate the effects of the add-on therapy. The analysis indicated significant differences in improvement of the PSV ( +4.15 vs. $-1.27 \mathrm{~cm} / \mathrm{sec})$, the EDV $(+1.83$ vs. $-0.84 \mathrm{~cm} / \mathrm{sec})$ and RI (-0.04 vs. +0.01 , respectively) between the add-on and control groups. No marked difference was observed between the change in renal volume over the 2-year study $\left(+3.29\right.$ vs. $\left.+5.06 \mathrm{~cm}^{3}\right)$. The ultrasonographic results indicate that the co-administration of berberine resulted in improved renal hemodynamics compared with the control therapy alone.

However, the results of the present study do not indicate that the baseline control treatment exerted no renoprotective effects. The less marked renoprotective effects observed in the control group may be explained by the following: i) Subjects had received the background treatment for years, and so its renoprotective effects may have reached the maximum; ii) only patients whose UACR was not adequately regulated by the background treatment were enrolled, and primary data of the parameters prior to the background treatment was unavailable; therefore, it was not possible to compare the parameters before and after the background treatment; iii) the disease progressed with the extension of the disease course.

Collectively, the baseline therapy and the co-administration of berberine were able to adequately control blood pressure, FPG and $\mathrm{HbA}_{1 \mathrm{c}}$ levels in patients with combined hypertension and T2DM. Despite exhibiting similar effects 
on blood pressure and blood glucose, the co-administration of berberine as an add-on therapy appeared to provide more effective renoprotection and renal hemodynamic improvement, in addition to inhibiting inflammation and oxidative stress in hypertensive patients with T2DM. Further studies are required to investigate the effects of berberine in hypertensive patients with T2DM that do not match the inclusion criteria of the current study, and the effects of berberine on other cardiovascular organs.

\section{References}

1. Wang M, Zhang W, Zhou Y and Zhou X: Association between serum angiotensin-converting enzyme 2 levels and postoperative myocardial infarction following coronary artery bypass grafting. Exp Ther Med 7: 1721-1727, 2014.

2. Chen G, McAlister FA, Walker RL, Hemmelgarn BR and Campbell NR: Cardiovascular outcomes in Framingham participants with diabetes: The importance of blood pressure. Hypertension 57: 891-897, 2011.

3. No authors listed: Hypertension in Diabetes study (HDS): II. increased risk of cardiovascular complications in hypertensive type 2 diabetic patients. J Hypertens 11: 319-325, 1993.

4. Tamura T, Said S, Harris J, Lu W and Gerdes AM: Reverse remodeling of cardiac myocyte hypertrophy in hypertension and failure by targeting of the renin-angiotensin system. Circulation 102: 253-259, 2000.

5. Takatsu M, Hattori T, Murase T, Ohtake M, Kato M, Nashima K, Nakashima C, Takahashi K, Ito H, Niinuma K, Aritomi S, et al: Comparison of the effects of cilnidipine and amlodipine on cardiac remodeling and diastolic dysfunction in Dahl salt-sensitive rats. J Hypertens 30: 1845-1855, 2012.

6. Yin M, Westenbrink B, Meissner M, van Gilst WH and de Boer RA: Variable effects of anti-diabetic drugs in animal models of myocardial ischemia and remodeling: A translational perspective for the cardiologist. Int J Cardiol 169: 385-393, 2013 .

7. Csiszar A, Toth J, Peti-Peterdi J and Ungvari Z: The aging kidney: Role of endothelial oxidative stress and inflammation. Acta Physiol Hung 94: 107-115, 2007.

8. Liu L, Liu Y, Qi B, Wu Q, Li Y and Wang Z: Nicorandil attenuates endothelial VCAM-1 expression via thioredoxin production in diabetic rats induced by streptozotocin. Mol Med Rep 9: 2227-2232, 2014.

9. Suzuki H, Sakamoto M, Hayashi T, Iuchi H, Ohashi K, Isaka T, Sakamoto N, Kayama Y, Tojo K, Yoshimura M and Utsunomiya K: Effects of co-administration of candesartan with pioglitazone on inflammatory parameters in hypertensive patients with type 2 diabetes mellitus: A preliminary report. Cardiovasc Diabetol 12: 71, 2013.

10. Baradaran A, Nasri H and Rafieian-Kopaei M: Oxidative stress and hypertension: Possibility of hypertension therapy with antioxidants. J Res Med Sci 19: 358-367, 2014.

11. Pan HZ, Zhang L, Guo MY, Sui H, Li H, Wu WH, Qu NQ, Liang $\mathrm{MH}$ and Chang D: The oxidative stress status in diabetes mellitus and diabetic nephropathy. Acta Diabetol 47 (Suppl 1): 71-76, 2010.

12. Nakamura A, Shikata K, Nakatou T, Kitamura T, Kajitani N, Ogawa D and Makino H: Combination therapy with an angiotensin-converting-enzyme inhibitor and an angiotensin II receptor antagonist ameliorates microinflammation and oxidative stress in patients with diabetic nephropathy. J Diabetes Investig 4: 195-201, 2013.

13. Sato-Horiguchi C, Ogawa D, Wada J, Tachibana H, Kodera R, Eguchi J, Nakatsuka A, Terami N, Shikata K and Makino H: Telmisartan attenuates diabetic nephropathy by suppressing oxidative stress in db/db mice. Nephron Exp Nephrol 121: e97-e108, 2012.

14. Guo Y, Liu Y and Wang Y: Beneficial effect of lycopene on anti-diabetic nephropathy through diminishing inflammatory response and oxidative stress. Food Funct 6: 1150-1156, 2015.

15. Ji X, Naito Y, Weng H, Ma X, Endo K, Kito N, Yanagawa N, Yu Y, Li J and Iwai N: Renoprotective mechanisms of pirfenidone in hypertension-induced renal injury: Through anti-fibrotic and anti-oxidative stress pathways. Biomed Res 34: 309-319, 2013.
16. Molavi B, Rassouli N, Bagwe S and Rasouli N: A review of thiazolidinediones and metformin in the treatment of type 2 diabetes with focus on cardiovascular complications. Vasc Health Risk Manag 3: 967-973, 2007.

17. Zhou G, Cheung AK, Liu X and Huang Y: Valsartan slows the progression of diabetic nephropathy in $\mathrm{db} / \mathrm{db}$ mice via a reduction in podocyte injury, and renal oxidative stress and inflammation. Clin Sci (Lond) 126: 707-720, 2014.

18. Luo Y, Yang SK, Zhou X, Wang M, Tang D, Liu FY, Sun L and Xiao L: Use of Ophiocordyceps sinensis (syn. Cordyceps sinensis) combined with angiotensin-converting enzyme inhibitors (ACEI)/ angiotensin receptor blockers (ARB) versus ACEI/ARB alone in the treatment of diabetic kidney disease: A meta-analysis. Ren Fail 37: 614-634, 2015.

19. Chandran G, Sirajudeen KN, Yusoff NS, Swamy M and Samarendra MS: Effect of the antihypertensive drug enalapril on oxidative stress markers and antioxidant enzymes in kidney of spontaneously hypertensive rat. Oxid Med Cell Longev 2014: 608512, 2014.

20. Kim J, Shon E, Kim CS and Kim JS: Renal podocyte injury in a rat model of type 2 diabetes is prevented by metformin. Exp Diabetes Res 2012: 210821, 2012.

21. Alhaider AA, Korashy HM, Sayed-Ahmed MM, Mobark M, Kfoury H and Mansour MA: Metformin attenuates streptozotocin-induced diabetic nephropathy in rats through modulation of oxidative stress genes expression. Chem Biol Interact 192: 233-242, 2011.

22. Drzewoski J and Zurawska-Klis M: Effect of gliclazide modified release on adiponectin, interleukin- 6 , and tumor necrosis factoralpha plasma levels in individuals with type 2 diabetes mellitus. Curr Med Res Opin 22: 1921-1926, 2002.

23. Zhang SQ, Sun YT, Xu TH, Zhang XF, Liu YZ, Ma MJ, Wang LN and Yao L: Protective effect of metformin on renal injury of C57BL/6J mouse treated with high fat diet. Pharmazie 69: 904-908, 2014.

24. Li MH, Zhang YJ, Yu YH, Yang SH, Iqbal J, Mi QY, Li B, Wang ZM, Mao WX, Xie HG and Chen SL: Berberine improves pressure overload-induced cardiac hypertrophy and dysfunction through enhanced autophagy. Eur J Pharmacol 728: 67-76, 2014.

25. Zhao HP, Hong Y, Xie JD, Xie XR, Wang J and Fan JB: Effect of berberine on left ventricular remodeling in renovascular hypertensive rats. Yao Xue Xue Bao 42: 336-341, 2007 (In Chinese).

26. Hong Y, Hui SC, Chan TY and Hou JY: Effect of berberine on regression of pressure-overload induced cardiac hypertrophy in rats. Am J Chin Med 30: 589-599, 2002.

27. Cheng F, Wang Y, Li J, Su C, Wu F, Xia WH, Yang Z, Yu BB, Qiu YX and Tao J: Berberine improves endothelial function by reducing endothelial microparticles-mediated oxidative stress in humans. Int J Cardiol 167: 936-942, 2013.

28. Li Z, Geng YN, Jiang JD and Kong WJ: Antioxidant and anti-inflammatory activities of berberine in the treatment of diabetes mellitus. Evid Based Complement Alternat Med 2014: 289264, 2014.

29. Chang W, Chen L and Hatch GM: Berberine as a therapy for type 2 diabetes and its complications: From mechanism of action to clinical studies. Biochem Cell Biol 2014 Dec 1: 1-8 [Epub ahead of print].

30. Bhutada P, Mundhada Y, Bansod K, Tawari S, Patil S, Dixit P, Umathe S and Mundhada D: Protection of cholinergic and antioxidant system contributes to the effect ofberberine ameliorating memory dysfunction in rat model of streptozotocin-induced diabetes. Behav Brain Res 220: 30-41, 2011.

31. Moghaddam HK, Baluchnejadmojarad T, Roghani M, Khaksari M, Norouzi P, Ahooie M and Mahboobi F: Berberine ameliorates oxidative stress and astrogliosis in the hippocampus of STZ-induced diabetic rats. Mol Neurobiol 49:820-826, 2014.

32. Ni WJ, Ding HH and Tang LQ: Berberine as a promising anti-diabetic nephropathy drug: An analysis of its effects and mechanisms. Eur J Pharmacol 760: 103-112, 2015.

33. Alter ML, Kretschmer A, Von Websky K, Tsuprykov O, Reichetzeder C, Simon A, Stasch JP and Hocher B: Early urinary and plasma biomarkers for experimental diabetic nephropathy. Clin Lab 58: 659-671, 2012.

34. Chen Q, He F, Feng X, Luo Z, Zhang J, Zhang L, Wang Y and Tong J: Correlation of Doppler parameters with renal pathology: A study of 992 patients. Exp Ther Med 7: 439-442, 2014.

35. Crutchley TA, Pearce JD, Craven TE, Stafford JM, Edwards MS and Hansen KJ: Clinical utility of the resistive index in atherosclerotic renovascular disease. J Vasc Surg 49: 148-155.e3, 2009. 
36. Nosadini R, Velussi M, Brocco E, Abaterusso C, Carraro A, Piarulli F, Morgia G, Satta A, Faedda R, Abhyankar A, Luthman $\mathrm{H}$ and Tonolo $\mathrm{G}$ : Increased renal arterial resistance predicts the course of renal function in type 2 diabetes with microalbuminuria. Diabetes 55: 234-239, 2006.

37. Hou YJ, Zhao YY, Xiong B, Cui XS, Kim NH, Xu YX and Sun SC: Mycotoxin-containing diet causes oxidative stress in the mouse. PLoS One 8: e60374, 2013.

38. Zhang M, Feng L, Gu J, Ma L, Qin D, Wu C and Jia X: The attenuation of Moutan Cortex on oxidative stress for renal injury in AGEs-induced mesangial cell dysfunction and streptozotocin-induced diabetic nephropathy rats. Oxid Med Cell Longev 2014: 463815, 2014.

39. Kawai T, Kamide K, Onishi M, Yamamoto-Hanasaki H, Baba Y, Hongyo K, Shimaoka I, Tatara Y, Takeya Y, Ohishi M and Rakugi H: Usefulness of the resistive index in renal Doppler ultrasonography as an indicator of vascular damage in patients with risks of atherosclerosis. Nephrol Dial Transplant 26: 3256-3262, 2011.

40. Alderman MH, Cohen $\mathrm{H}$ and Madhavan S: Diabetes and cardiovascular events in hypertensive patients. Hypertension 33 : 1130-1134, 1999.

41. Impellizzeri D, Esposito E, Attley J and Cuzzocrea S: Targeting inflammation: New therapeutic approaches in chronic kidney disease (CKD). Pharmacol Res 81: 91-102, 2014.

42. Tripepi G, Mallamaci F and Zoccali C: Inflammation markers, adhesion molecules and all-cause and cardiovascular mortality in patients with ESRD: Searching for the best risk marker by multivariate modeling. J Am Soc Nephrol 16 (Suppl 1): 83-88, 2005.

43. Lee HJ, Jeong KH, Kim YG, Moon JY, Lee SH, Ihm CG, Sung JY and Lee TW: Febuxostat ameliorates diabetic renal injury in a streptozotocin-induced diabetic rat model. Am J Nephrol 40: 56-63, 2014.

44. Bae EH, Kim IJ, Joo SY, Kim EY, Choi JS, Kim CS, Ma SK, Lee J and Kim SW: Renoprotective effects of the direct renin inhibitor aliskiren on gentamicin-induced nephrotoxicity in rats. J Renin Angiotensin Aldosterone Syst 15: 348-361, 2014.

45. Šilhavý J, Zídek V, Landa V, Šimáková M, Mlejnek P, Oliyarnyk O, Malínská H, Kazdová L, Mancini M and Pravenec M: Rosuvastatin ameliorates inflammation, renal fat accumulation, and kidney injury in transgenic spontaneously hypertensive rats expressing human $\mathrm{C}$-reactive protein. Physiol Res 64: 295-301, 2015.
46. Park JW, Bae EH, Kim IJ, Ma SK, Choi C, Lee J and Kim SW: Renoprotective effects of paricalcitol on gentamicin-induced kidney injury in rats. Am J Physiol Renal Physiol 298: F301-F313, 2010.

47. Shibata R, Sato K, Pimentel DR, Takemura Y, Kihara S, Ohashi K, Funahashi T, Ouchi N and Walsh K: Adiponectin protects against myocardial ischemia reperfusion injury through AMPK- and COX-2-dependent mechanisms. Nat Med 11: 1096-1103, 2005.

48. Oliveira CS, Saddi-Rosa P, Crispim F, Canani LH, Gerchman F, Giuffrida FM, Vieira JG, Velho G and Reis AF: Association of ADIPOQ variants, total and high molecular weight adiponectin levels with coronary artery disease in diabetic and non-diabetic Brazilian subjects. J Diabetes Complications 26: 94-98, 2012.

49. Kopf S, Oikonomou D, von Eynatten M, Kieser M, Zdunek D, Hess G, Morcos M, Forsblom C, Bierhaus A, Groop PH, Nawroth PP and Humpert PM: Urinary excretion of high molecular weight adiponectin is an independent predictor of decline of renal function in type 2 diabetes. Acta Diabetol 51: 479-489, 2014.

50. Wu YH, Chuang SY, Hong WC, Lai YJ, Chang GJ and Pang JH: Berberine reduces leukocyte adhesion to LPS-stimulated endothelial cells and VCAM-1 expression both in vivo and in vitro. Int J Immunopathol Pharmacol 25: 741-750, 2012.

51. Choo BK and Roh SS: Berberine protects against esophageal mucosal damage in reflux esophagitis by suppressing proinflammatory cytokines. Exp Ther Med 6: 663-670, 2013.

52. Liu WH, Hei ZQ, Nie H, Tang FT, Huang HQ, Li XJ, Deng YH, Chen SR, Guo FF, Huang WG, Chen FY and Liu PQ: Berberine ameliorates renal injury in streptozotocin-induced diabetic rats by suppression of both oxidative stress and aldose reductase. Chin Med J (Engl) 121: 706-712, 2008.

53. Wu D, Wen W, Qi CL, Zhao RX, Lü JH, Zhong CY and Chen YY: Ameliorative effect of berberine on renal damage in rats with diabetes induced by high-fat diet and streptozotocin. Phytomedicine 19: 712-718, 2012. 